Subscriber access provided by Caltech Library

\title{
Article
}

\section{QM-Mechanism-Based Hierarchical High-Throughput in silico Screening Catalyst Design for Ammonia Synthesis}

Qi An, Yidi Shen, Alessandro Fortunelli, and William A. Goddard

J. Am. Chem. Soc., Just Accepted Manuscript • DOI: 10.1021/jacs.8b10499 • Publication Date (Web): 27 Nov 2018

Downloaded from http://pubs.acs.org on November 27, 2018

\section{Just Accepted}

"Just Accepted" manuscripts have been peer-reviewed and accepted for publication. They are posted online prior to technical editing, formatting for publication and author proofing. The American Chemical Society provides "Just Accepted" as a service to the research community to expedite the dissemination of scientific material as soon as possible after acceptance. "Just Accepted" manuscripts appear in full in PDF format accompanied by an HTML abstract. "Just Accepted" manuscripts have been fully peer reviewed, but should not be considered the official version of record. They are citable by the Digital Object Identifier (DOI®). "Just Accepted" is an optional service offered to authors. Therefore, the "Just Accepted" Web site may not include all articles that will be published in the journal. After a manuscript is technically edited and formatted, it will be removed from the "Just Accepted" Web site and published as an ASAP article. Note that technical editing may introduce minor changes to the manuscript text and/or graphics which could affect content, and all legal disclaimers and ethical guidelines that apply to the journal pertain. ACS cannot be held responsible for errors or consequences arising from the use of information contained in these "Just Accepted" manuscripts. 


\title{
QM-Mechanism-Based Hierarchical High-Throughput in silico Screening Catalyst Design for Ammonia Synthesis
}

\author{
Qi An, ${ }^{1,2, *}$ Yidi Shen ${ }^{2}$, Alessandro Fortunelli, ${ }^{1,3,}$, and William A. Goddard III ${ }^{1, *}$ \\ ${ }^{1}$ Materials and Process Simulation Center (MSC), California Institute of Technology, \\ Pasadena, California 91125, United States \\ 2 Department of Chemical and Materials Engineering, University of Nevada-Reno, \\ Reno, Nevada 89577, United States \\ ${ }^{3}$ CNR-ICCOM, Consiglio Nazionale delle Ricerche, ThC2-Lab, Pisa 56124, Italy \\ *Corresponding authors’ E-mails: qia@unr.edu, alessandro.fortunelli@cnr.it,wag@caltech.edu

\begin{abstract}
We propose and test a hierarchical high-throughput screening (HHTS) approach to catalyst design for complex catalytic reaction systems that is based on quantum-mechanics (QM) derived full reaction networks with QM rate constants, but simplified to examine only the reaction steps likely to be rate-determining. We illustrate this approach by applying it to determine the optimum dopants (our of 35 candidates) to improve the turn-over-frequency (TOF) for the Fe-based Haber-Bosch ammonia-synthesis process. We start from the QM-based free-energy reaction network for this reaction over $\mathrm{Fe}(111)$ which contains the 26 most important surface configurations and 17 transition states at operating conditions of temperature and pressure, from which we select the key reaction steps that might become rate-determining for the alloy. These are arranged hierarchically by decreasing free-energy reaction barriers. We then extract from the full reaction network a reduced set of reaction rates required to quickly predict the effect of the catalyst changes on each barrier. This allows us to test new candidates with only $1 \%$ of the effort for a full calculation. Thus we were able to quickly screen 34 candidate dopants to select a small subset ( $\mathrm{Rh}, \mathrm{Pt}, \mathrm{Pd}, \mathrm{Cu})$ that satisfy all criteria, including stability. Then from these four candidates expected to increase the TOF for $\mathrm{NH}_{3}$ production, we selected the best candidate (Rh) for a more complete free-energy and kinetic analysis (10 times the effort for HHTS but still $10 \%$ of the effort for a complete analysis of the full reaction network). We predict that $\mathrm{Rh}$ doping of $\mathrm{Fe}$ will increase the $\mathrm{TOF}$ for $\mathrm{NH}_{3}$ synthesis by a factor of $\sim 3.3$ times compared to $\mathbf{F e}(111)$, in excellent agreement with our HHTS predictions, validating this approach.
\end{abstract}




\section{Introduction}

In order to significantly accelerate the rate of discovery and optimization of novel catalyst systems required to meet the urgent problems in energy and environment faced by society ${ }^{1,2}$, we need to employ quantum-mechanics (QM) based rational design (in silico optimization) so that experiment can focus on the most promising candidates. Previously, a great deal of progress on simpler catalytic processes has been made by using the Sabatier principle ${ }^{3}$ as translated into volcano curve modeling, ${ }^{4,5}$ which has provided useful guidelines and general trends to help identify promising candidates for improving simple catalysts. But this analysis is based on a simplified scenario of the catalysis process, essentially presenting one key ratedetermining step. ${ }^{6,7}$ In contrast, the mechanisms involved in many realistic, industriallyrelevant catalytic processes are often extremely complex with many competing pathways.

Fortunately QM computational methods are becoming sophisticated and powerful enough to successfully master this level of complexity. Thus we recently reported a complete analysis of the Haber-Bosch (HB) synthesis of $\mathrm{NH}_{3}$ based on $\mathrm{Fe}$ catalysts at high pressure and temperature. ${ }^{8}$ Here we considered the most efficient Fe(111) surface for the HB reactions. ${ }^{8}$ This required full quantum-mechanics-based free-energy calculations of the 26 most important surface configurations at operating conditions of temperature and pressure and including the free energy based reaction rates between these species. This was followed by full kinetic Monte Carlo analysis for 45 minutes, allowing the ensemble of configurations to come to steady state at reaction conditions. The final Turn-over-Frequency (TOF) of $17.7 / \mathrm{sec}$ per site for the $\mathrm{Fe}(111)$ single crystal at $400 \mathrm{C}$ and $20 \mathrm{~atm}$ compares well with the experimental $\mathrm{TOF}=9.7 / \mathrm{sec}$ per site. This excellent agreement is a testament to the accuracy of the PBE-D3 QM, to the completeness of the reaction network, and to the robustness of the kinetic Monte Carlo (kMC) predictions. However, such a complete set of QM calculations takes an enormous effort in personnel, computer resources, and time to completion, and is not practical to use on 10's to 1000's of possibilities.

To make rapid progress in dramatically accelerating our discovery of improved catalysts, we need to be able to quickly estimate the TOF of new candidates, say at $1 \%$ of the effort for a full 
analysis, and then to validate the most promising cases with a more compete but still fast and reliable procedure, say at $10 \%$ of the effort for a full calculation. We present here this general procedure which we illustrate by applications to discover binary alloys to improve the HB process. We aim to achieve sufficiently quantitative accuracy that the experiments can examine just the few candidates we identify as most promising and focus on optimizing process and operating conditions of these selected cases.

Here we propose the hierarchical protocol for rapid high-throughput screening (HHTS), which we demonstrate by application to discovering improved catalysts for energy intensive HB ammonia synthesis ${ }^{9-12}$. Our experimentally validated full reaction network for the HB process ${ }^{8}$ at industrial conditions, identified 10 potentially rate determining steps in the full network, which we partition here into 4 distinct and diverse processes that might most likely become rate-determining as we dope the Fe catalyst:

(i) activation of the N-N bond (itself composed of 4 different steps, from adsorption from the gas phase to interconversion between different adsorption modes),

(ii) hydrogenation of $\mathrm{NH}_{\mathrm{x}}$-adsorbed species (itself also distinguished into 3 different steps with $\mathrm{x}=0-2$ ),

(iii) desorption of the $\mathrm{NH}_{3}$ product (in 2 different points along the catalytic path),

(iv) poisoning of catalytically active sites by reactant $\left(\mathrm{H}_{2}\right)$ or product $\left(\mathrm{NH}_{3}\right)$ species.

All 10 of these distinct steps could be rate-determining. As the first step, we use our full kMC kinetic analysis to simplify this reaction network to single out the minimum number of processes (4) and corresponding reaction free energies required to estimate the overall catalytic rate with minimum computational effort but simultaneously with sufficient accuracy to avoid missing any potential candidates. This reduces the computational effort to $1 \%$ of the full QM calculation, making it practical to examine a large set (34) of dopants that might accelerate the rates by reducing the barriers for all of the 4 distinct reaction steps most likely to become ratedetermining. From these 34 alloying elements our protocol discovers 4 that are very promising to significantly accelerate $\mathrm{HB}$ rates with respect to the pure Fe catalyst: $\mathrm{Rh}, \mathrm{Pt}, \mathrm{Pd}$ and $\mathrm{Cu}$, all of which are novel and not yet tested experimentally. 
Then as the next step for in silico design, we select the case (Rh) predicted to be most promising and compute an extended (but still not the full set) of QM-based free-energy rates that we use in $\mathrm{kMC}$ simulations to predict the $\mathrm{NH}_{3}$ production rate. This extended calculation requires $\sim 10 \%$ of the full calculation. We predict that the Rh doped system will have a TOF 4.7 times faster than the pure $\mathrm{Fe}(111)$ system, in excellent agreement with HHTS expectations, thus validating our approach. This HHTS process provides a breakthrough advance, with quantitative measure of how much improvement can be obtained, to trigger experiments focused on optimizing reaction conditions, knowing that an improved performance is guaranteed.

\section{The hierarchical high-throughput screening (HHTS) approach}

As starting points of our HHTS approach, we assume to have available:

(i) a complete free-energy network (for $\mathrm{Fe}(111) \mathrm{HB}$ this includes 26 surface configurations) for the target catalytic reaction, including reaction rates between them (17 for this case); and (ii) a strategy to change the catalyst composition over a chosen set of possibilities.

To test our approach, (i), we use the free-energy reaction network for ammonia synthesis (HB) process over $\mathrm{Fe}(111)$ derived in Ref.8, in which density-functional theory (DFT) predictions ${ }^{13-}$ 16 free energies and reaction rates used the $(2 \times 2)$ unit cell of Fe(111) (PBE-D3) ${ }^{13,14}$ exchangecorrelation functional, see Ref.8 and the Supplementary Information, SI, for computational details). This is one of the most extensive first-principles-based investigation of a heterogeneous catalytic reaction ever reported. This free-energy reaction network, evaluated at $673 \mathrm{~K}, \mathrm{P}\left(\mathrm{H}_{2}\right)=15 \mathrm{~atm}, \mathrm{P}\left(\mathrm{N}_{2}\right)=5 \mathrm{~atm}, \mathrm{P}\left(\mathrm{NH}_{3}\right)=1 \mathrm{~atm}$, is shown as a linear diagram in Figure 1. The predicted Turn-Over-Frequency (TOF) of $17.7 \mathrm{NH}_{3} / \mathrm{sec}$ for our $2 \times 2$ computational cell is in excellent agreement with the TOF $=9.7 \mathrm{NH}_{3} / \mathrm{sec}$ from single crystal experiments ${ }^{8}$ (changing the barrier for the rate determining step by $0.04 \mathrm{eV}$, would reduce the predicted rate to 9.7 showing the sensitivity of TOF to the reaction barriers). 
The energy diagram shown Fig.1 suggests that the barrier for $\mathrm{N}_{2}$ absorption/desorption is the highest. But the kinetic Monte Carlo analysis show that the dissociative chemisorption of $\mathrm{H}_{2}$ and the desorption of $\mathrm{NH}_{3}$ play an essential role in providing the empty sites required for the $\mathrm{N}_{2}$ to bind and as the $\mathrm{NN}$ bonds are reduced from 3 to 0 . As a result, the desorption of $\mathrm{NH}_{3}$ is also rate limiting for $\mathrm{Fe}(111)$. Additional potential RDSs involve the successive addition of $\mathrm{H}^{*}$ to $\mathrm{NH}_{\mathrm{x}}{ }^{*}$ via Langmuir-Hinshelwood (LH) additions, leading to 4 possible RDSs, that also depend on $\mathrm{H}_{2}$ chemisorption and $\mathrm{NH}_{3}$ desorption of $\mathrm{H}$. Thus, there are at least 10 potential RDS, each of which may require specific types of sites.

The strategy (ii), we adopt to modify the Fe(111) catalyst is to replace one of the 4 topmost Fe atoms in the $(2 \times 2)$ unit cell with one from a set of 34 metal elements covering a large portion of the periodic table (substitutional surface doping, see Figure 2-A,B, with the set of dopants shown in Figure 2-C). We consider as dopants the 29 transition metal elements, plus selected lanthanides for a total of 34 elements (alkalis such as $\mathrm{K}$ are not considered since they adopt non-substitutional configurations, other main group metal and non-metal elements will be considered in future work).

Our HHTS protocol then consists of the following steps:

(a) analyze the free-energy diagram to single out the largest barriers and arrange them in decreasing order;

(b) define a simple criterion to estimate the effect of the proposed change in the catalyst on each barrier;

(c) evaluate each criterion in sequence in the order of decreasing barrier over the set of candidates, at each step restricting only to candidates which have passed previous (higherlevel) screening by leading to a decrease in the overall barrier, thus sequentially sifting out potential candidates;

(d) test the stability of the proposed catalyst change with respect to possible degradation mechanisms; 
(e) for candidates which have survived all previous criteria, reconstruct a significant portion of the free-energy diagram and perform explicit kinetic Monte Carlo (kMC) simulations to predict actual increase in catalytic efficiency.

We now discuss these steps in more detail.

Step (a) is illustrated in Figure 1, in which the largest barriers are assigned different colors to make the analysis clearer. We use the Dijkstra's algorithm ${ }^{17}$ to single out the shortest (minimum-barrier) path between initial and final states within the given reaction network, which gives the largest contribution to the rate constant under steady-state conditions. ${ }^{18} \mathrm{We}$ then section this path into a sequence of lowest-free-energy resting states and highest-freeenergy transition states, in which the resting states are local minima of the free-energy profile while transition states are the highest-free-energy points between two resting states. The freeenergy difference between each couple of transition and resting states defines our set of freeenergy barriers, which we then arrange in decreasing order. It can be noted in this connection that, for the largest barrier, its resting state exhibits the most negative degree of rate control (DRC) index and its transition state exhibits the most positive DRC index as defined by Campbell et al., ${ }^{19}$ but the second-largest and lower barriers typically exhibit negligible DRC indexes. We note that the free-energy diagram and thus the associated hierarchy of reaction barriers depend also on the experimental conditions, i.e., temperature and pressure of reactants and products. Here we select for definitiveness $\mathrm{T}=673 \mathrm{~K}, \mathrm{P}\left(\mathrm{H}_{2}\right)=15 \mathrm{~atm}, \mathrm{P}\left(\mathrm{N}_{2}\right)=5 \mathrm{~atm}$, and $\mathrm{P}\left(\mathrm{NH}_{3}\right)=1 \mathrm{~atm}$. The ideal goal of current research on $\mathrm{HB}$ is to drastically reduce the extreme conditions of industrial ammonia synthesis (HB) process, typically held at 773-823 K and total pressure of 150-250 atm, by reducing temperature by $100-150 \mathrm{~K}$ and pressures by a factor of 10. This first step has analogies with and has been inspired by previous work, in particular the concept of DRC in catalysis ${ }^{19}$, i.e., the analysis of the overall catalytic process to single out which are the rate-determining mechanistic steps on which effort should be concentrated to achieve acceleration.

The previous volcano approaches ${ }^{5,6}$ typically assume linear scaling in the reaction rates to focus on a specific rate-determining step (RDS) and identify catalysts that improve (accelerate) this 
step or to reconstruct the energy diagram on given catalysts so as to identify the optimum catalyst, while the DRC approach could use a combination of RDSs depending on the DRC indexes but only focuses on steps that are relevant for a specific catalyst. ${ }^{19}$ Therefore, these approaches provide a fast catalyst screening for reactions that have relatively simple mechanisms. In contrast, HHTS considers that there may be a number of potential RDSs, so that improving one of them may lead to a very different RDS. Thus, we arrange all the potentially rate-determining steps of any complex reaction mechanism in hierarchical order, and then we sequentially filter candidate catalysts that accelerate the overall reaction rate also when the largest barrier corresponding to the previous RDS is no longer rate determining. For example in $\mathrm{NH}_{3}$ synthesis over $\mathrm{Fe}(111)$ we found 4 distinct steps in the $\mathrm{N}_{2}$ reduction, three distinct steps in adding $\mathrm{H}^{*}$ to $\mathrm{NH}_{\mathrm{x}}{ }^{*}$ and two distinct $\mathrm{NH}_{3}$ desorption steps. Based on the relative barriers we reduced this to two potential RDS for $\mathrm{N}_{2}$ desorption, one for $\mathrm{H}^{*}$ plus $\mathrm{NH}_{\mathrm{x}}$, and one $\mathrm{NH}_{3}$ desorption. This is especially important when optimization approaches the ideal catalyst presenting a uniform energy landscape, in which all the potentially rate-determining steps exhibit similar barriers and similar rates. Thus, HHTS must consider a diversity of energetic and barrier calculations. This makes HHTS much more likely to succeed for a much wider and rigorous applicability. The only assumption in HHTS is that the dopants can change the relative energies of the configurations and therefore the kinetics, but do not significantly change the overall reaction mechanism.

In step (b), for each barrier we define a simple criterion to estimate how the given barrier will be affected by a change in the catalyst. We do so by associating a reaction to each barrier and using the corresponding electronic reaction energy (same DFT approach and VASP software ${ }^{20}$ as in Ref.8) to estimate the change in free-energy barrier. In other words, we explicitly calculate only the free-energy of reference states. In general, the free-energy of transition states can be explicitly calculated or estimated via Brønsted-Evans-Polanyi (BEP) relations. ${ }^{4,5,19}$ But we found that the key barriers for $\mathrm{N}_{2}$ adsorption and hydrogen migration are essentially constant (independent of the dopant), which corresponds to assuming unitary slope BEP linear relationship between free-energy barrier and reaction free energies. ${ }^{21-23}$ Of course, for the predicted best dopant, Rh, we actually calculate the barriers. The good correspondence of this 
full predicted with the in-silico estimates justifies the approximation. Simple DFT geometry relaxations are needed to evaluate such criteria for each element. Figure 3 illustrates the configurations used in the screening procedure, as discussed in detail in the next Section.

Step (c) defines our hierarchical or sequential systematic screening procedure, in which we use the estimated barriers from step (b) to sift out candidates expectedly leading to a decrease in the overall barrier. This step is illustrated as an Eratosthenes' sieve or Wenn diagram in Figure 2 and Figure S1 of the SI. We apply the first criterion, corresponding to the largest barrier, to the complete set of 34 dopant elements. We apply the second criterion only to the elements that have passed the first criterion showing the potential to decrease the overall barrier. We apply the third criterion only to the elements that have passed the first and second criteria, and so on. Restricting lower-level criteria only to candidates that have passed higher-level ones significantly reduces the total computational effort.

As for step (d), we test one common catalyst degradation mechanism for alloy systems, i.e., inverse segregation of the dopant from the surface into the bulk of the catalyst. Clearly, other catalyst degradation mechanisms are possible. E.g., some dopants are expected to vaporize as hydrides under the given conditions so we have not included these in the set of dopants (e.g., As transforming into $\mathrm{AsH}_{3}$ ), while others may aggregate into clusters, etc. Other degradation mechanisms could be considered after defining a chemical potential for competing species or mechanisms, but we do not consider them in this proof-of-principle study.

In the final step (e), for the most promising candidate which has survived all screening criteria (i.e., Rhodium, Rh) we evaluate entropic terms and explicit energy barriers for steps that have proved to be important to kinetics ${ }^{8}$. In this way we check that our rapid-screening estimates hold, and we quantitatively predict the expected increase in catalytic efficiency.

\section{In silico strategy for doped $\mathrm{Fe}(111)$ catalysts for HB synthesis}

The previous QM studies for $\mathrm{HB}$ on $\mathrm{Fe}(111)$ found 4 important reaction barriers that could potentially become rate determining. Two are involved in adsorbing/dissociating $\mathrm{N}_{2}$, one is involved in $\mathrm{H}_{\mathrm{ad}}$ reacting with $\mathrm{NH}_{\mathrm{x}}$ to form $\mathrm{NH}_{\mathrm{x}+1}$, one is associated with $\mathrm{H}_{2}$ poisoning, and one 
involves $\mathrm{NH}_{3}$ adsorption/desorption. But in analyzing the rates using $\mathrm{kMC}$ these rates can be related back to fundamental differences in free energies of various intermediates which allows us to mimimize the states that must be analyzed.

\section{Criterion 1. Triple bonded $\mathrm{N}_{2}$ absorption over $2 \mathrm{~N}$ state}

The largest free-energy barrier in the diagram of Figure 1 - yellow line, barrier (1) - is associated with $\mathrm{N}_{2}$ adsorption over the $2 \mathrm{~N}$ state. The system first transforms from its lowenergy resting state with a high coverage of $\mathrm{NH}_{\mathrm{y}}$-absorbed species into the $2 \mathrm{~N}$ [zig-zag] configuration (Figure 3A), which has two nearest-neighbor vacant bridge sites so that it can dissociate $\mathrm{N}_{2}$, which is followed by $\mathrm{N}_{2}$ adsorption and dissociation. We recall ${ }^{8}$ that the $2 \mathrm{~N}$ [zigzag] configuration is named "zig-zag" because an alternative "linear" configuration with the same stoichiometry exists, as illustrated in Figure S2 $(a, b)$ of the SI (the same alternative applies to configurations with different stoichiometry such as $2 \mathrm{~N}_{-} \mathrm{NH}_{2} \mathrm{H}, 2 \mathrm{~N}_{-} \mathrm{N}_{2}[\gamma]$, etc.). A high energy price is associated with generating the 2N[zig-zag] configuration on $\mathrm{Fe}(111)$. An improved catalyst should decrease this energy price and thus the stability of $\mathrm{N}$ adatoms on the surface, while still exhibiting a sufficiently large affinity to nitrogen to be able to dissociate $\mathrm{N}_{2}$ effectively. We identify our first criterion, connected with the $\mathrm{N}_{2}$ adsorption/dissociation barrier, with the electronic reaction energy from the 2N_NH${ }_{2} \mathrm{H}$ [zig-zag] (Figure 3 B-D) resting state to $2 \mathrm{~N}$ [zig-zag]: "2N_ $\mathrm{NH}_{2} \mathrm{H}_{\text {[zig-zag] }} \rightarrow 2 \mathrm{~N}$ [zig-zag] $+\mathrm{NH}_{3}$ " (see Figure 3 for pictorial illustrations). Note that the highest saddle point for subsequent $\mathrm{N}_{2}$ dissociation is 0.3 $\mathrm{eV}$ below the saddle point corresponding to barrier(1). This might become rate determining when the $\mathrm{N}_{2}$ absorption energy onto the catalyst surface is too small. We thus complement our first criterion by evaluating the $\mathrm{N}_{2}$ adsorption energy on $2 \mathrm{~N}$ [zig-zag], i.e., the energy difference between the " $2 \mathrm{~N}$ [zig-zag] $+\mathrm{N}_{2}$ gas-phase" and $2 \mathrm{~N}_{-} \mathrm{N}_{2}[\gamma$,zig-zag] configurations, and ensuring that this adsorption energy is $>0.5 \mathrm{eV}$ as an additional constraint. We finally estimate barrier(1) via the formulae:

$\operatorname{barrier}(1)=\Delta \mathrm{E}\left\{2 \mathrm{~N} \_\mathrm{NH}_{2} \_\mathrm{H}[\right.$ zig-zag $] \rightarrow 2 \mathrm{~N}[$ zig-zag $\left.]+\mathrm{NH}_{3}\right\}-0.104 \mathrm{eV}$

constraint : $\Delta \mathrm{E}\left\{2 \mathrm{~N} \_\mathrm{N}_{2}[\gamma, \mathrm{zig}\right.$-zag $] \rightarrow 2 \mathrm{~N}[$ zig-zag $\left.]+\mathrm{N}_{2}\right\}>0.5 \mathrm{eV}$ 
where 2N_NH ${ }_{2}$ H[zig-zag], 2N[zig-zag], and 2N_N $\mathrm{N}_{2}[\gamma, \mathrm{zig}-\mathrm{zag}$ ] (Figure $3 \mathrm{M}-\mathrm{O}$ ) correspond to the surface configurations discussed above, $\mathrm{N}_{2}$ and $\mathrm{NH}_{3}$ refer to molecules in the gas phases, and " $\Delta \mathrm{E}$ " refers to the electronic energy difference between the states after arrow and states before arrow. The energy value " $0.104 \mathrm{eV}$ " was computed based on the free energy corrections of the pure Fe(111) surface. The barrier (1) corresponds to the energy barrier (1) values in Figure 1 for the pure $\mathrm{Fe}(111)$ surface. All the electronic energies of the doped configurations are listed in the xsl file in the SI.

\section{Criterion 2. $\mathrm{H}_{2}$ poisoning $\mathrm{N}_{2}$ dissociation via the $2 \mathrm{~N}_{-} 2 \mathrm{H}$ resting state}

The second highest barrier in Figure 1 - blue line, barrier(2) - is associated with the same saddle point, but is connected with the observation that the 2N_2H[linear] (Figure $3 \mathrm{E}-\mathrm{F}$ ) configuration can represent a resting state under some conditions (e.g. at low $\mathrm{NH}_{3}$ pressure) which can slow down catalysis $\left(\mathrm{H}_{2}\right.$ poisoning). Therefore, the second criterion is expressed by the formula:

barrier $(2)=\Delta \mathrm{E}\left\{2 \mathrm{~N} \_2 \mathrm{H}[\right.$ linear2 $] \rightarrow 2 \mathrm{~N}[$ zig-zag $\left.]+\mathrm{H}_{2}\right\}+0.113 \mathrm{eV}$

where 2N_2H[linear2] corresponds to the surface configuration (Figure S2) discussed above, and $\mathrm{H}_{2}$ refers to hydrogen in the gas phase. The energy value " $0.113 \mathrm{eV}$ " was computed based on the free energy corrections of the pure Fe(111) surface.

\section{Criterion 3. $\mathrm{H}_{\text {ad }}$ migration to $\mathrm{NH}_{2 a d}$ to form $\mathrm{NH}_{3 a d}$}

The conversion of adsorbed $\mathrm{N}$ into $\mathrm{NH}_{\mathrm{y}}$ species is also associated with high energy barriers, the third largest in the free-energy diagram of Figure 1 - green line, barrier(3) -, and our third criterion estimates these barriers. We focus on the highest-barrier hydrogenation (or hydrogen migration) mechanistic step, which is the hydrogenation of $2 \mathrm{~N}_{-} \mathrm{NH}_{2} \mathrm{H}$ : “2N_NH $\mathrm{NH}_{2} \mathrm{H} \rightarrow 2 \mathrm{~N} \_\mathrm{NH}_{3}$ ". We use the Brønsted-Evans-Polanyi principle and assume that the energy barrier of this step will be a linear function of the energy difference between $2 \mathrm{~N}_{-} \mathrm{NH}_{2} \mathrm{H}$ and $2 \mathrm{~N} \_\mathrm{NH}_{3} \mathrm{H}$ configurations (Figure $3 \mathrm{G}-\mathrm{H}$ ), thus estimating the third barrier with the formula:

$\operatorname{barrier}(3)=\Delta \mathrm{E}\left\{2 \mathrm{~N} \_\mathrm{NH}_{2} \_\mathrm{H}[\right.$ zig-zag $]+1 / 2 \mathrm{H}_{2} \rightarrow 2 \mathrm{~N}_{-} \mathrm{NH}_{3} \_\mathrm{H}[$ zig-zag $\left.]\right\}+1.549 \mathrm{eV}(4)$ 
where the 2N_NH $\mathrm{NH}_{3} \mathrm{H}$ [zig-zag] corresponds to the surface configruation discussed above. The energy value "1.549 $\mathrm{eV}$ " was computed based on the free energy corrections of the pure $\mathrm{Fe}(111)$ surface.

Criterion 4. $\mathrm{NH}_{3}$ desorption. Finally, $\mathrm{NH}_{3}$ desorption can be rate-limiting - red line, barrier(4) - , so the fourth criterion ensures that a high $\mathrm{NH}_{3}$ desorption energy does not decrease in the overall rate. The largest $\mathrm{NH}_{3}$ desorption barrier is expected close to the $2 \mathrm{~N}$ [zig-zag] state, for the $2 \mathrm{~N} \_\mathrm{NH}_{3} \mathrm{H}[\mathrm{zig}-\mathrm{zag}]$ configuration, and is estimated with respect to the $2 \mathrm{~N}_{-} \mathrm{NH}_{2} \mathrm{H}$ [zigzag] resting state as follows:

barrier(4) $=\Delta \mathrm{E}\left\{2 \mathrm{~N} \_\mathrm{NH}_{2} \_\mathrm{H}[\mathrm{zig}-\mathrm{zag}]+1 / 2 \mathrm{H}_{2} \rightarrow 2 \mathrm{~N} \_\mathrm{NH}_{3}\right.$ H[zig-zag $\left.]\right\}+\Delta \mathrm{H}\left\{2 \mathrm{~N} \_\mathrm{NH}_{3} \mathrm{H}[\mathrm{zig}-\right.$ zag] $\rightarrow$ 2N_H[zig-zag] $\left.+\mathrm{NH}_{3}\right\}+0.425 \mathrm{eV}$

where 2N_H[zig-zag] (Figure $3 \mathrm{~K}-\mathrm{L}$ ) corresponds to the surface configruation discussed above. The energy value " $0.425 \mathrm{eV}$ " was computed based on the free energy corrections of the pure $\mathrm{Fe}(111)$ surface.

\section{Criterion 5. Preference of dopant in top layer versus second layer}

The above criteria assume that the overall free-energy diagram is only altered quantitatively, not qualitatively, by the proposed change in the catalyst. The fifth and last criterion tests that the catalyst change does not introduce qualitative alterations of the diagram, i.e., the insurgence of degradation mechanisms. Inverse segregation of the dopant element into the bulk is a common mechanism for dopants that reduces the interaction of the catalyst surface with $\mathrm{NH}_{\mathrm{y}^{-}}$ adsorbed species. ${ }^{24} \mathrm{We}$ evaluate this degradation mechanism only for the $4 \mathrm{~N}$ configuration (Figure 3I) and for the dopant going from the top surface layer into the subsurface layer (Figure 3J). We estimate the increase in the previous barriers as follows:

$\operatorname{barrier}(1-4)=\operatorname{barrier}(1-4)+\max \{\Delta \mathrm{E}\{4 \mathrm{~N}[$ subsurface-dopant $] \rightarrow 4 \mathrm{~N}[$ surface-dopant $]\}, 0\}$

where $4 \mathrm{~N}$ [subsurface-dopant] and $4 \mathrm{~N}$ [surface-dopant] correspond to the configurations discussed above, "max" refers to the maximum between the two numbers in the bracket, and "barrier(1-4)" refers to the the maxium barrier for steps 1 to 4 . 


\section{Application of hierarchical in silico screening to 34 dopants.}

The application of the rapid in silico screening to HB over singly-top-surface-substitutionallydoped $\mathrm{Fe}(111)$ is illustrated in Figures 2 and S1 using the original free energy diagram. ${ }^{8}$ The electronic energy results of the five criteria are listed in Table 1 (with a complete report in the xsl file in the SI).

\section{Criterion 1. $\mathrm{N}_{2}$ desorption/dissociation}

Of the 34 dopants, only 12 led to a barrier lower than the $1.68 \mathrm{eV}$ for $\mathrm{Fe}$, but we kept an additional 3 that were only a little above 1.68, as shown in Table 1 . The other 19 cases were not examined further. Of these 19, 14 are more electropostivie than $\mathrm{Fe}$ (electronegativity $(\chi)=1.8$ ), while Mo is the same and $\mathrm{Tc}, \mathrm{Re}$, and $\mathrm{Hg}$ (all $\chi=1.9$ ) are only slightly more electronegative. This indicates that alloying a more electronegative element helps with $\mathrm{N}_{2}$ dissociation. Thus the elimination of Os $(\chi=2.2)$ is the only outliner. It is interesting that Haber found that pure Os does do $\mathrm{NH}_{3}$ synthesis. Of these 12 selected dopants, none violate the constraint equation (2).

We note here that the best two, $\mathrm{Rh}$ and $\mathrm{Pd}$, with a barrer lower by $0.17 \mathrm{eV}$ might lead to a rate increase by a factor of 18.75 for our target conditions.

\section{Criterion 2. $\mathrm{H}_{2}$ poisoning to the $\mathrm{N}_{2}$ adsorption}

Here we eliminate 6 more. This includes the 4 closed shell elements (Cd, Au, Ag, and $\mathrm{Zn}$ ). This suggests that we want open-shell d-electrons for good $\mathrm{N}_{2}$ dissociation barriers. It is not obvious why Ir and Ru have a high barrier. It is interesting that pure Ru (hcp crystal structure) does do $\mathrm{NH}_{3}$ synthesis.

\section{Criterion 3. $\mathrm{NH}_{x}$ hydrogenation}

All 6 remaining candidates are better than Fe.

\section{Criterion $4 . \mathrm{NH}_{3}$ desorption}

All 6 remaining candidates are better than Fe. It is important to note that criterion $\mathrm{NH}_{3}$ desorption becomes an important step for some (e.g. Pt-) doped systems. Note that these steps 
would not be considered within a DRC approach because of their low DRC indexes on the undoped catalyst.

\section{Criterion 5. Surface stability}

Here two cases: Co and Ni, prefer subsurface. This may be because they are slightly smaller than Fe.

\section{The Winners}

We are left with four winners: Rh and Pt predicted to be 4 times better than pure Fe and Pd and $\mathrm{Cu}$ predicted to be 2 times better than pure $\mathrm{Fe}$.

$\mathrm{Rh}$ and Pt lead to a reduction in the overall barrier of $0.06 \mathrm{eV}$ : hydrogen poisoning limits the potential reduction of 0.17 or $0.13 \mathrm{eV}$ according to criterion (1). Reducing the overall barrier by $0.06 \mathrm{eV}$ would not allow dramatically less extreme industrial conditions, but - if realized should guarantee a reduction by a factor of $\sim 4$ in the energy consumption even maintaining the same conditions ( $\approx 200 \mathrm{~atm}$ total pressure and $773-823 \mathrm{~K}$ temperature) and industrial plants as used presently. Further improvements can likely be achieved by implementing multiple doping strategies. The HHTS-estimated $\mathrm{NH}_{3}$ production rates for optimal dopants are also listed in Table 1.

As discussed in Ref.8, the barriers associated with these phenomena are also intimately related to the charge and magnetic state of surface atoms, as we confirm by reporting an analysis of the changes in charges and spins of surface atoms upon doping, illustrated in Figure S3.

\section{Full QM analysis and Kinetic Study of Rh-Doping}

The above in silico analysis focused on 11 key states that determine the major barriers. This allowed us to reduce the candidates from 34 to 4 . Now we will consider the full analysis for one of the winners, Rh, which we estimated would be 4 times better than Fe. To this end we extend the study from the 11 configurations considered above and consider the 21 configurations and 13 barriers most important for the kinetics (Figure S4 and S5 of SI). 
We performed QM simulations on a (2x2) unit cell of the $\mathrm{Fe}(111)$ surface substitutionally doped in the top layer with one $\mathrm{Rh}$ atom and reconstructed a substantial portion of the reaction energy diagram, as shown in Figure 4. A simplified reaction pathway is illustrated pictorially in Figure S4 showing the surface structure step by step, to clarify the nature of each adsorption site and the interactions between these adsorbed species. The favorable mechanistic paths are the same as on the pure Fe surface. ${ }^{8}$ Figure 4 plots a standard state free energy diagram which does not include the configurational entropy of adsorbates. ${ }^{25}$ However, configurational effects are taken into account in our kMC simulations model by including the configuration counting into the rate constants.

To validate our assumptions, we compare the 4 key reaction steps used in our HHTS approach between pure $\mathrm{Fe}$ and $\mathrm{Rh}$-doped catalysts. For the pure Fe catalyst, the free energy barriers for N2 adsorption (step1), H2-poisoning (step2), H migration (step3), and NH3 desorption (step4) are $1.68,1.57,1.52$, and $1.43 \mathrm{eV}$, respectively. Based on our assumptions, the estimated barriers from HHTS for Rh-based catalyst are 1.51, 1.60, 1.31 and $1.44 \mathrm{eV}$ for these 4 steps, respectively, whereas explicit calculations on the Rh-doped catalyst give values of: 1.44, 1.62, 1.31 and $1.35 \mathrm{eV}$, respectively. The difference between estimated and explicit calculations is within a maximum error of $0.09 \mathrm{eV}$, thus validating our free-energy barrier estimates.

We used the energetics from the free-energy diagrams of Figures 1 and 4 as input to kinetic Monte Carlo (kMC) simulations, using the same set of 21 configurations and 13 barriers for both pure and $\mathrm{Rh}$-doped $\mathrm{Fe}(111)$.

On pure $\mathrm{Fe}(111)$, this leads to production of $2441 \mathrm{NH}_{3}$ molecules produced by our $(2 \times 2)$ unit cell, corresponding to a predicted TOF $=4.6 \mathrm{NH}_{3} / \mathrm{sec}$ per $(2 \times 2)$ site, which can be compared to $\mathrm{TOF}=3.68$ for our simplified model

On Rh-doped $\mathrm{Fe}(111)$, this leads to production of $35980 \mathrm{NH}_{3}$ molecules produced by our $(2 \times$ 2) unit cell, corresponding to a predicted TOF $=9.7 \mathrm{NH}_{3} / \mathrm{sec}$ per $(2 \times 2)$ site, which can be compared to 14.6 in our simplified model.

However, the Rh-doped system can be further improved by exploiting its sensitivity to $\mathrm{H}_{2}$ poisoning and working in a lean- $\mathrm{H}_{2}$ régime. Thus reducing the $\mathrm{H}_{2}$ pressure from 15 to $6.5 \mathrm{~atm}$ : 
under conditions of $\mathrm{T}=673 \mathrm{~K}, \mathrm{P}\left(\mathrm{H}_{2}\right)=6.5 \mathrm{~atm}, \mathrm{P}\left(\mathrm{N}_{2}\right)=5 \mathrm{~atm}$, and $\mathrm{P}\left(\mathrm{NH}_{3}\right)=1 \mathrm{~atm}$, we predict TOF $=15.3 \mathrm{NH}_{3} / \mathrm{sec}$ per $(2 \times 2)$ site, which is a factor of 3.3 larger than on $\mathrm{Fe}(111)$, in good agreement with expectations from the HHTS estimates. Thus we estimate that Rh doped Fe might lead to an overall TOF that is $\sim 3.3$ times the current Fe based catalysts. We note here that reducing $\mathrm{H}_{2}$ pressure slows down the reaction rate for $\mathrm{Fe}$, but increases it for $\mathrm{Rh}$. Given the high cost of $\mathrm{H}_{2}$, working under lean- $\mathrm{H}_{2}$ conditions may be beneficial in terms of production costs.

Finally, to provide information on mechanistic details, the steady-state apparent $\Delta \mathrm{G}$ (i.e., the logarithm of the relative populations or residence times) for the most important states are reported in Table $\mathrm{S} 1$ for both pure and doped $\mathrm{Fe}(111)$.

\section{Modifications of the PBE-D3(BJ) free-energy diagram}

Any HTS approach depends on the accuracy of the assumed free-energy diagram. The one employed here was derived using the DFT/PBE-D3 method, ${ }^{8}$ and leads to excellent agreement with experimental ammonia production rates at low $\mathrm{NH}_{3}$ pressure ${ }^{26}$ (theory: $17.7 / \mathrm{sec}$ for a $2 \times 2$ surface cell; experiment 9.7). We now test how robust our analysis is with respect to a change in the system energetics. In particular, one issue of Figure 1 is that the overall free-energy change $(\delta \mathrm{G})$ for the ammonia synthesis reaction $\left[\mathrm{N}_{2}+3 \mathrm{H}_{2} \rightarrow 2 \mathrm{NH}_{3}\right]$ is predicted by PBED3(BJ) to be $-0.54 \mathrm{eV}$ at $673^{\circ} \mathrm{K}$, and 5:15:1 atm of $\mathrm{N}_{2}, \mathrm{H}_{2}$ and $\mathrm{NH}_{3}$, respectively. In contrast the experimental value is $\delta \mathrm{G}=-0.06 \mathrm{eV}$ under the same conditions. ${ }^{27}$ To test how sensitive the HHTS predictions are to this issue, we use an empirical correction ${ }^{28}$ and modify the free-energy diagram of Figure 1 by adding a quantity of $[0.08 \cdot \mathrm{y}] \mathrm{eV}$ to the free energy of $\mathrm{NH}_{\mathrm{y}}$ surface species. This corresponds to assuming that PBE-D3(BJ) overestimates the N-H vs. N-Fe bond strength by this quantity (for transition states corresponding to hydrogenation mechanisms we use half of the $0.08 \mathrm{eV}$ correction). The overall gas-phase energetics of the HB reaction is so recovered by distributing the PBE-D3(BJ) error in $\delta G$ uniformly over the energetics of surface species. The free-energy diagram resulting after applying these corrections is reported in Figure S6 of the SI, under the same conditions of Figure 1: T $=673 \mathrm{~K}, \mathrm{P}\left(\mathrm{H}_{2}\right)=15 \mathrm{~atm}, \mathrm{P}\left(\mathrm{N}_{2}\right)=5 \mathrm{~atm}$, 
$\mathrm{P}\left(\mathrm{NH}_{3}\right)=1$ atm (thermodynamic equilibrium conversion using experimental energetics corresponds to 1.7 atm $\mathrm{NH}_{3}$ pressure, thus $\mathrm{P}\left(\mathrm{NH}_{3}\right)=1$ atm is consistent with roughly $50 \%$ conversion typically used in the industrial HB process). The five basic criteria singled out in Section 3.a need to be only slightly modified after applying these corrections (the main difference being that the resting state of the system is now the $4 \mathrm{~N}$ configuration), and are detailed in the SI.

We applied the high-throughput screening protocol using these new criteria. Interestingly, the 5 elements ( $\mathrm{Rh}, \mathrm{Pt}, \mathrm{Cu}, \mathrm{Pd}$, with the addition of $\mathrm{Ni}$ which is roughly identical to $\mathrm{Fe}$ ) which are suggested as promising using the free-energy diagram of Figure 1 are still present in the final HHTS set derived using the diagram of Figure S6, as illustrated in Figure S7. The major difference is that:

(1) the $\mathrm{NH}_{3}$ production rate at $\mathrm{P}\left(\mathrm{NH}_{3}\right)=1$ atm by the $\mathrm{Fe}(111)$ surface is decreased (the production rate at low $\mathrm{NH}_{3}$ pressure using Figure $\mathrm{S} 6$ does not change much and is also consistent with the experiment ${ }^{23}$ ),

(2) therefore, the expected acceleration due to doping is increased, and

(3) more elements are included in the set of potentially promising dopants, such as $\mathrm{Zn}, \mathrm{Ag}, \mathrm{Au}$, Cd. In particular Zn seems particularly appealing due to its small size mismatch with Fe.

Apart from calling for a proper experimental validation of the accuracy of DFT for this system, these finding suggests that, overall, the set of optimal dopants determined in Figure 2 is reasonably robust to a change in the theoretical method. Much larger enhancements in production rates are predicted when using the free energy diagram of Figure S6. The expected maximum in catalytic activity is realized for $\mathrm{Cu}, \mathrm{Ni}$, and $\mathrm{Pd}$, with a reduction in overall barrier amounting to $0.35-0.37 \mathrm{eV}$, thus translating into a potential speed-up by more than factor of 100 in $\mathrm{HB}$ process rate.

To conclude, we note that, clearly, several variants of HHTS can be conceived. For example, the kinetic model can be analyzed and solved via other methods not considered in this work, such as micro-kinetic modeling as in the DRC approach. ${ }^{19,29}$ In this connection, note that, to implement our hierarchical approach, DRC indexes could be defined for each section of the 
free-energy diagram potentially leading to rate-determining steps. The screening criteria can be improved by explicit transition state calculations. The free-energy diagram can be made more accurate, e.g., by improving over the harmonic approximation to evaluate entropic contributions, using more accurate exchange-correlation functionals or higher-level computational methods, or adjusting empirical corrections to the energetics of different metals. ${ }^{30}$ Other strategies for changing the catalys ${ }^{31}$ such as multiple (ternary, etc.) doping can be investigated. Finally, catalytic selectivity rather than simple activity can be targeted for optimization. ${ }^{19}$

\section{Conclusions}

A goal of current research in theoretical materials science is to devise new computational approaches that can be appropriate to complex (and thus realistic) materials design, going beyond simple outlining of general trends towards the completion of a fully predictive theoretical modeling. In the catalysis field, this thrust faces significant challenges. First, the property to be optimized (catalytic activity) is connected with saddle points in the potential energy surface (PES) and corresponding energy barriers, that intrinsically require a higher computational effort with respect to description of local minima and equilibrium states. Second, the free energy diagram of a realistic catalytic mechanism is often so complex and with so many competing paths that the behavior of activity or selectivity in terms of any given descriptor cannot be represented with a single volcano curve, i.e., the property/variable function becomes so rugged that its optimum value is to be found in a pocket or niche of this fragmented free-energy landscape, at the intersection of several Brønsted-Evans-Polanyi (BEP) energy relationships. In these realistic cases, high-throughput screening seems the only viable path to predictive rational catalyst design.

Here we answer these challenges by developing a hierarchical high-throughput screening (HHTS) protocol for rapid in silico design of novel catalysts, and demonstrate its usefulness by applying it to the ammonia synthesis (Haber-Bosch) process over doped Fe(111) surface. The approach starts from a complete free-energy diagram of the given catalytic reaction, which is 
then analyzed to single out and arrange the barriers of key mechanistic steps in hierarchical order, defining for each of these steps a simple energy criterion for deciding whether a proposed change in the catalyst composition will lead to an increase or a decrease of the corresponding barrier, and sequentially screening a set of doping elements according to these criteria, to finally arrive at a subset of promising candidates satisfying all criteria. Promising candidates can then be tested by reconstructing larger portions of the free-energy diagram followed by kMC simulations of steady-state production rates (100 time the effort). Application of this protocol to ammonia synthesis over doped Fe(111) surface identifies a set of promising dopants: $\mathrm{Rh}, \mathrm{Pt}, \mathrm{Cu}, \mathrm{Pd}$, that might dramatically improve the TOF compared to the current Fe catalysts. Most of these canditates are quite novel, providing a set for experimental confirmation. We followed this prediction from HHTS that Rh doping of Fe might significantly enhance $\mathrm{NH}_{3} \mathrm{TOF}$, with complete calculations including all 44 surface configurations, free energy reaction barriers and full kMC to predict that $\mathbf{R h}$ doping of $\mathbf{F e}$ might increase the TOF by $\sim 3.3$ times compared to pure Fe. This provides a focused target for experimental confirmation, that may potentially reduce in HB energy consumption by several fold, even with the same conditions and industrial plants as used presently.

Future developments include variants of the screening criteria, refinement of the free-energy diagram analysis via micro-kinetic model techniques, ${ }^{19,29}$ adjustments of empirical corrections to the energetics of different metals, ${ }^{30}$ or other strategies for changing the catalyst, ${ }^{31}$ such as multiple (ternary, etc.) doping.

\section{ASSOCIATED CONTENT}

\section{Supporting Information}

The Supporting Information is available free of charge on the ACS Publications website at DOI:xxx. 
Computational details, in silico strategy for doped Fe(111) catalysts for HB synthesis, KMC simulations details, and energy diagram of Rh-doped Fe catalyst.

Figures include Alternative illustration of the screening protocol; "Linear" vs. "zig-zag" $2 \mathrm{~N}$ configurations for the $2 \mathrm{~N}$ system; Atomic charges and spins for selected configuration; Surface structure for reaction steps in Figure 4; $\mathrm{N}_{2}$ dissociation steps on Rh-doped $\mathrm{Fe}(111)$ surface; DFT/PBE-D3 free energy diagram including semi-empirical correction; and Barriers for promising dopants estimated (A) without or (B) including empirical corrections.

Xlsx files with all raw energy data.

\section{Acknowledgements}

This work was supported by the U.S. Department of Energy (USDOE), Office of Energy Efficiency and Renewable Energy (EERE), Advanced Manufacturing Office Next Generation R\&D Projects under contract no. DE-AC07-05ID14517 (program manager Dickson Ozokwelu, in collaboration with Idaho National Laboratories, Rebecca Fushimi). Q.A. receives the support from American Chemical Society Petroleum Research Fund (PRF\# 58754-DNI6). A.F. gratefully acknowledges financial support from a Short-Term Mission (STM) funded by Italian Consiglio Nazionale delle Ricerche (CNR). We would like to thank the Information Technology department at the University of Nevada, Reno for computing time on the High Performance Computing Cluster (Pronghorn). Some calculations were also carried out on a GPU-cluster provided by DURIP (Cliff Bedford, program manager).

\section{Competing interests}

The authors declare no competing interests. 


\section{References}

(1) Jones, D. J.; Gibson, V. C.; Green, S. M.; Maddox, P. J.; White, A. J. P.; Williams, D. J. Discovery and optimization of new chromium catalysts for ethylene oligomerization and polymerization aided by high-throughput screening. J. Am. Chem. Soc. 2005, 127 (31), 11037-11046.

(2) Chen, B.; Parker, G. II; Han, J.; Meyyappan, M.; Cassell, A. M. Heterogeneous singlewalled carbon nanotube catalyst discovery and optimization. Chem. Mater. 2012, 14 (4), 1891-1896.

(3) Sabatier, P. Hydrogenations et deshydrogenations par catalyse. Ber. Dtsch. Chem. Ges. 1911, 44 (3), 1984-2001.

(4) Nørskov, J. K.; Bligaard, T.; Rossmeisl, J.; Christensen, C. H. Towards the computational design of solid catalysts. Nature Chem. 2009, 1, 37-46.

(5) Jacobsen, C. J. H.; Dahl, S.; Clausen, B. S.; Bahn, S.; Logadottir, A.; Nørskov, J. K. Catalyst design by interpolation in the periodic table: bimetallic ammonia synthesis catalysts. J. Am. Chem. Soc. 2001, 123 (34), 8404-8405.

(6) Ferrin, P.; Simonetti, D.; Kandio, S.; Kunkes, E.; Dumesic, J.A.; Nørskov, J.K.; Mavrikakis, M. Modeling ethanol decomposition on transition metals: a combined application of scaling and Brønsted-Evans-Polanyi relations. J. Am. Chem. Soc. 2009, 131 (16), 5809-5815.

(7) Greeley, J.; Jaramillo, T. F.; Bonde, J.; Chorkendorff, I.; Nørskov, J. K. Computational high-throughput screening of electrocatalytic materials for hydrogen evolution. Nature Mater. 2006, 5, 909-913.

(8) Qian, J.; An, Q.; Fortunelli, A.; Nelsen, R. S.; Goddard, W. A. III. Reaction mechanism and kinetics for ammonia synthesis on the Fe(111) surface. J. Am. Chem. Soc. 2018, 140 (20), 6288-6297.

(9) Erisman, J. W.; Sutton, M. A.; Galloway, J.; Klimont, Z.; Winiwarter, W. How a century of ammonia synthesis changed the world. Nat. Geosci. 2008, 1, 636-639. 
(10) Schlögl, R. Catalytic synthesis of ammonia—a "never-ending story"? Angew. Chem., Int. Ed. 2003, 42 (18), 2004-2008.

(11) Mittasch, A.; Frankenburg, W. Early studies of multicomponent catalysts. Adv. Catal. 1950, 2, 81-104.

(12) Hara, M.; Kitano, M.; Hosono, H. Ru-loaded C12A7: $\mathrm{e}^{-}$electride as a catalyst for ammonia synthesis. ACS Catal. 2017, 7 (4), 2313- 2324.

(13) Perdew, J. P.; Burke, K.; Ernzerhof, M. Generalized gradient approximation made simple. Phys. Rev. Lett. 1996, 77, 3865-3868.

(14) Perdew, J. P.; Burke, K.; Ernzerhof, M. Erratum: generalized gradient approximation made simple. Phys. Rev. Lett. 1997, 78, 1396-1396.

(15) Johnson, E. R.; Becke, A. D. A post-hartree-fock model of intermolecular interactions: inclusion of higher-order corrections. J. Chem. Phys. 2006, 124, 174104.

(16) Grimme, S.; Antony, J.; Ehrlich, S.; Krieg, H. A consistent and accurate ab initio parametrization of density functional dispersion correction (DFT-D) for the 94 elements HPu. J. Chem. Phys. 2010, 132, 154104.

(17) Dijkstra, E. W. A note on two problems in connexion with graphs. Numer. Math. 1959, $1,269-271$.

(18) Wales, D. J. Energy landscapes: calculating pathways and rates. Int. Rev. Phys. Chem. 2006, 25, 237-282.

(19) Wolcott, C. A.; Medford, A. J.; Studt, F.; Campbell, C. T. Degree of rate control approach to computational catalyst screening. J. Catal. 2015, 330, 197-207.

(20) Kresse, G.; Furthmüller, J. Efficient iterative schemes for ab initio total-energy calculations using a plane-wave basis set. Phys. Rev. B 1996, 54 (16), 11169-11186.

(21) Bronsted, N. Acid and basic catalysis. Chem. Rev. 1928, 5 (3), 231-338.

(22) Evans, M. G.; Polanyi, M. Inertia and driving force of chemical reactions. Trans. Faraday Soc. 1938, 34, 11-24. 
(23) Montemore, M. M.; Medlin, J. W. Scaling relations between adsorption energies for computational screening and design of catalysts. Catal. Sci. Technol. 2014, 4, 3748-3761.

(24) Mortensen, J. J.; Hansen, L. B.; Hammer, B.; Nørskov, J. K. Nitrogen adsorption and dissociation on $\mathrm{Fe}(111)$. J. Catal. 1999, 182 (2), 479-488.

(25) Campbell, C. T.; Sprowl, L. H.; Árnadóttir, L. Equilibrium constants and rate constants for adsorbates: two-dimensional (2D) ideal gas, 2D ideal lattice gas, and ideal hindered translator models J. Phys. Chem. C 2016, 120 (19), 10283-10297.

(26) Somorjai, G. A.; Materer, N. Surface structures in ammonia synthesis. Top. Catal. 1994, 1 (3-4), 215-231.

(27) Chase Jr., M. W.; Davies, C. A.; Downey, J. J. R.; Frurip, D. J.; McDonald, R. A.; Syverud, A. N. NIST-JANAF thermochemical tables, 4th ed.; Journal of Physical and Chemical Reference Data Monograph 9; American Chemical Society: Washington, DC, 1998.

(28) Hughes, T. F.; Friesner, R. A. Development of accurate DFT methods for computing redox potentials of transition metal complexes: results for model complexes and application to cytochrome. J. Chem. Theory Comput. 2012, 8 (2), 442-459.

(29) Li, L.; Sholl, D. S. Computational identification of descriptors for selectivity in syngas reactions on a $\mathrm{Mo}_{2} \mathrm{C}$ catalyst. ACS Catal. 2015, 5 (9), 5174-5185.

(30) Zaffran, J.; Michel, C.; Auneau, F.; Delbecq, F.; Sautet, P. Trade-off between accuracy and universality in linear energy relations for alcohol dehydrogenation on transition metals. J. Phys. Chem. C 2015, 119 (23), 12988-12998.

(31) Foster, S. L.; Perez Bakovic, S. I.; Duda, R. D.; Maheshwari, S.; Milton, R. D.; Minteer, S. D.; Janik, M. J.; Renner, J. N.; Greenlee, L. F. Catalysts for nitrogen reduction to ammonia. Nature Catal. 2018, 1, 490-500. 


\section{Figure Captions}

Figure 1. The stardard state free energy diagram at the DFT/PBE-D3 level for ammonia synthesis over a (2x2) unit cell of the $\mathrm{Fe}(111)$ surface, evaluated at $673 \mathrm{~K}, \mathrm{P}\left(\mathrm{H}_{2}\right)=15 \mathrm{~atm}$, $\mathrm{P}\left(\mathrm{N}_{2}\right)=5$ atm, $\mathrm{P}\left(\mathrm{NH}_{3}\right)=1$ atm. The barriers selected for high-throughput screening are highlighted in color and numbered. Black is the linear pathway and orange is the alternative pathway from the optimum reaction barriers. The notations for the adsorbed species represent the key configurations (Figure S4 of SI) along the reaction path. Free energies in eV.

Figure 2. (A,B) Schematic depictions of the (2x2) unit cell of $\mathrm{Fe}(111)$ both pure (A) and with one substitutional dopant (B). Bronze spheres represent the top layer, dark grey spheres represent the second layer, while white spheres are third layer Fe atoms. In (B) one dopant atom colored in purple replaces one topmost (bronze) Fe atom. (C) Portion of the periodic table selected for catalyst screening (34 elements). In the top row the screening criteria are indicated in colors, and the elements are also highlighted using the color of the criterion which has sifted them out.

Figure 3. Schematic depiction of the configurations used for reaction energies (the numbers are electronic energies (eV) from DFT simulations) for estimating barriers (energies for Rh-doping case). The $\mathrm{Rh}, \mathrm{N}$ and $\mathrm{H}$ atoms are represented by purple, blue and red balls, respectively. Bronze, dark and white spheres represent the top, the second, and the third layer Fe atoms, respectively. To represent the complexity of various doping sites, we use different symbols here for adsorbed species than Figure 1 and 4. The symbol (l) represents the linear $2 \mathrm{~N} \_2 \mathrm{H}\left[\right.$ linear2] structure and the c (or f) represents the doping close (or far) to the $\mathrm{N}, \mathrm{H}, \mathrm{NH}_{2}$ and $\mathrm{NH}_{3}$ species.

Figure 4. The stardard state energy landscape for $\mathrm{NH}_{3}$ synthesis reactions on $\mathrm{Rh}$-doped $\mathrm{Fe}(111)$ surface under $673 \mathrm{~K}$ and $20 \mathrm{~atm}$ conditions. The lowest energy state $3 \mathrm{~N}_{-} \mathrm{NH}_{2}$ is taken as reference, with a free energy of zero. Black is the linear pathway and orange is the alternative pathway from the optimum reaction barriers. The notations for the adsorbed species represent the key configurations (Figure S4 of SI) along the reaction path. To be consistent with Figure 1, we used the same symbols for adsorbed species. 


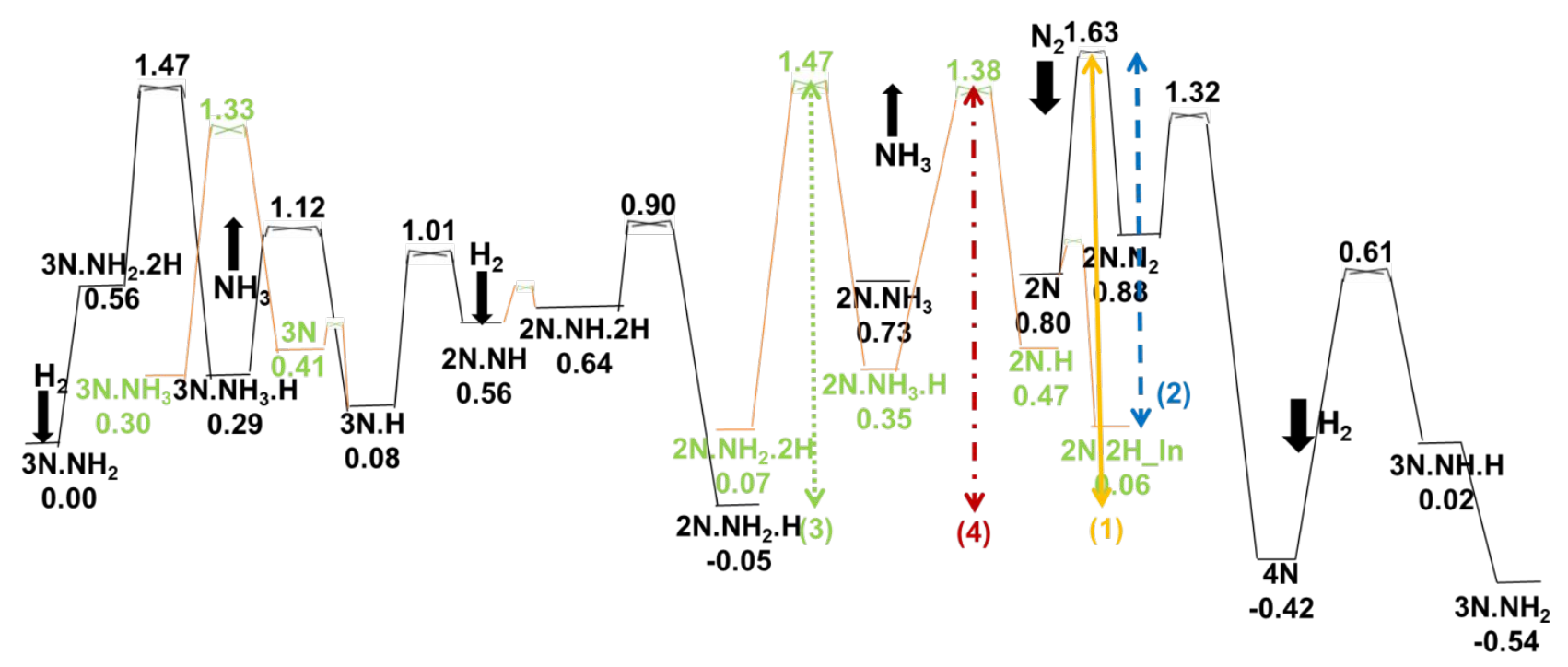

Figure 1. The stardard state free energy diagram at the DFT/PBE-D3 level for ammonia synthesis over a $(2 \times 2)$ unit cell of the $\mathrm{Fe}(111)$ surface, evaluated at $673 \mathrm{~K}, \mathrm{P}\left(\mathrm{H}_{2}\right)=15 \mathrm{~atm}$, $\mathrm{P}\left(\mathrm{N}_{2}\right)=5 \mathrm{~atm}, \mathrm{P}\left(\mathrm{NH}_{3}\right)=1$ atm. The barriers selected for high-throughput screening are highlighted in color and numbered. Black is the linear pathway and orange is the alternative pathway from the optimum reaction barriers. The notations for the adsorbed species represent the key configurations (Figure S4 of SI) along the reaction path. Free energies in eV. 


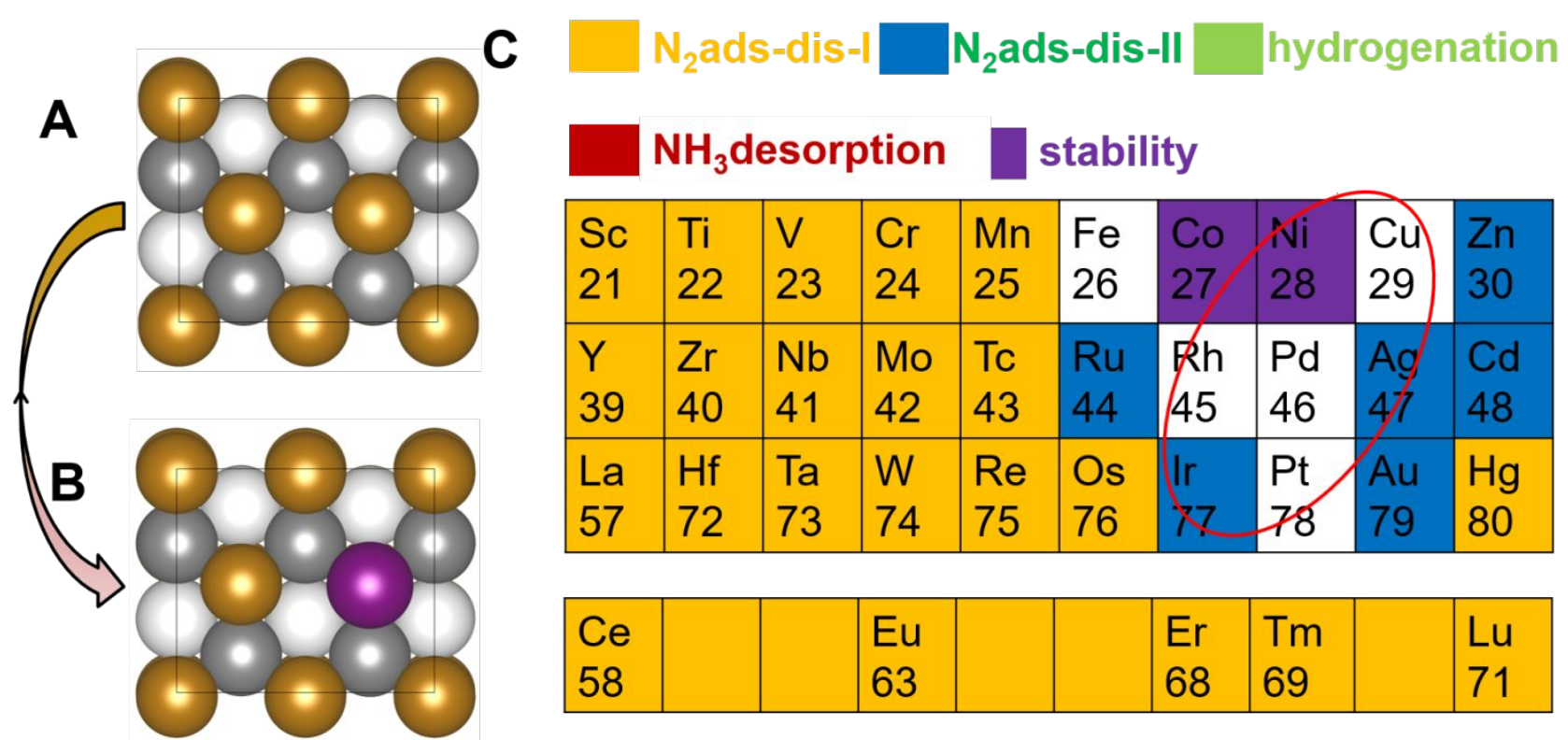

Figure 2. (A,B) Schematic depictions of the (2x2) unit cell of Fe(111) both pure (A) and with one substitutional dopant (B). Bronze spheres represent the top layer, dark grey spheres represent the second layer, while white spheres are third layer Fe atoms. In (B) one dopant atom colored in purple replaces one topmost (bronze) Fe atom. (C) Portion of the periodic table selected for catalyst screening (34 elements). In the top row the screening criteria are indicated in colors, and the elements are also highlighted using the color of the criterion which has sifted them out. 


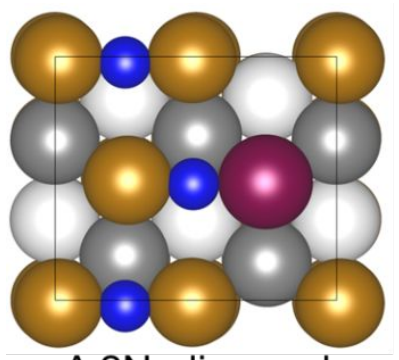

$$
\text { A 2N_diagonal }
$$

$-202.60$

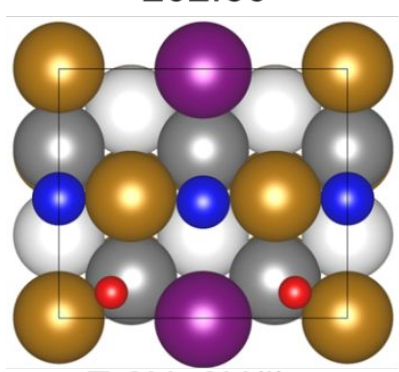

E 2N_2H(I)c

$-210.86$

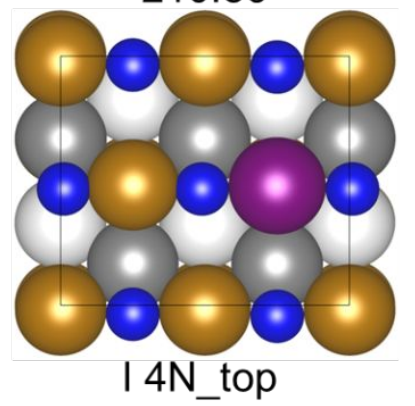

$-221.36$

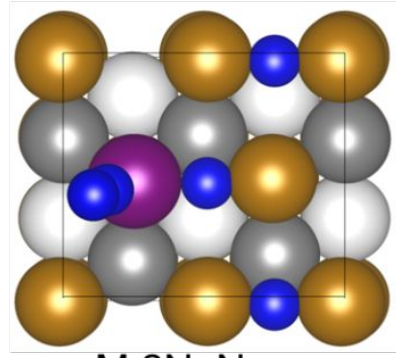

$\mathrm{M} 2 \mathrm{~N} \_\mathrm{N}_{2} \mathrm{C}$

$-219.99$

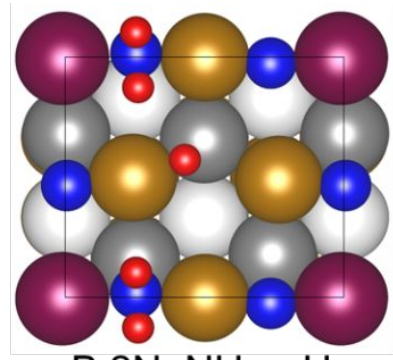

B 2N_NH $\mathrm{NH}_{2} \mathrm{C}$

$-223.62$

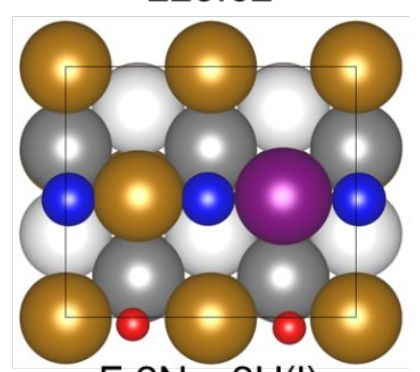

F 2Nc_2H(I)

$-210.73$

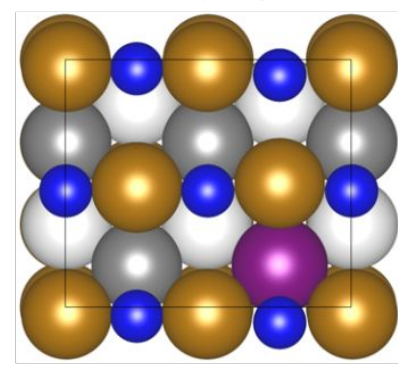

J 4N_sub

$-220.82$

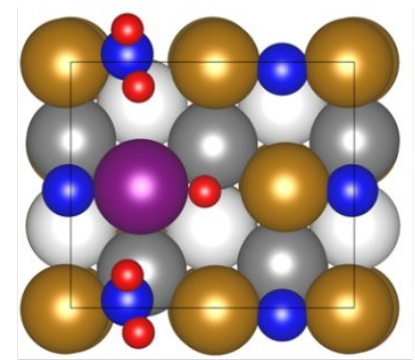

C $2 \mathrm{~N} \_\mathrm{NH}_{2} \mathrm{f}-\mathrm{H}$

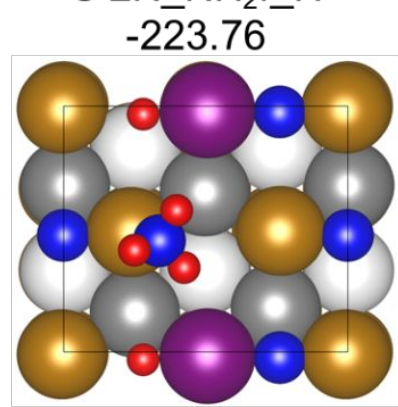

G 2N_ $\mathrm{NH}_{3} \mathrm{f} \_\mathrm{H}$

$-227.38$

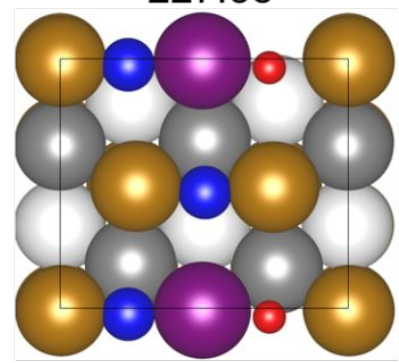

$\mathrm{K} 2 \mathrm{~N} \_\mathrm{Hc}$

$-206.61$

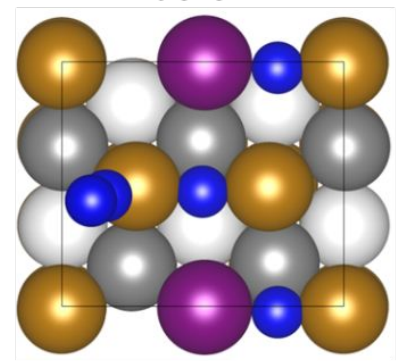

$\mathrm{N} 2 \mathrm{~N} \_\mathrm{N}_{2} \mathrm{ff}$

$-219.85$

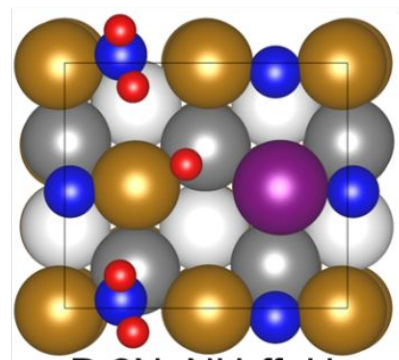

D 2N_NH $\mathrm{Nf}_{-} \mathrm{H}$ $-223.69$

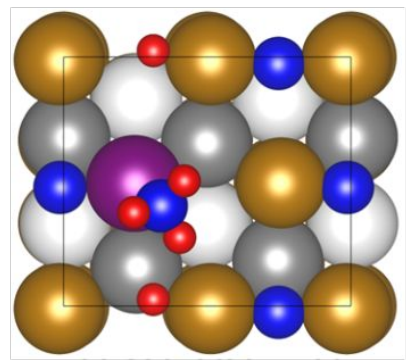

$\mathrm{H} 2 \mathrm{~N} \mathrm{NH}_{3} \mathrm{C}-\mathrm{H}$ $-227.09$

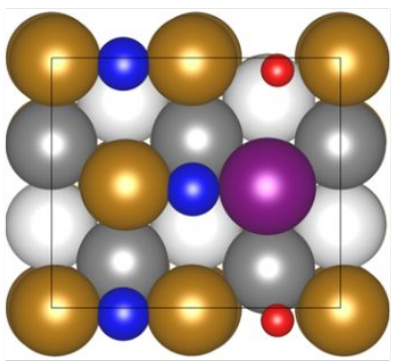

L 2Nc_H

$-206.52$

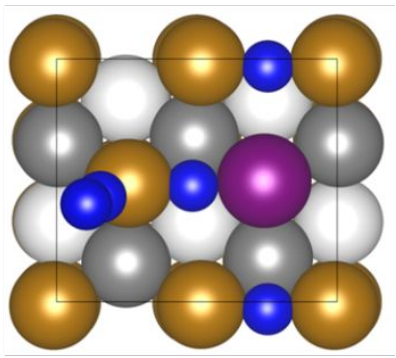

$\mathrm{O} 2 \mathrm{~N} \_\mathrm{N}_{2} \mathrm{f}$

$-219.86$

Figure 3. Schematic depiction of the configurations used for reaction energies (the numbers are electronic energies (eV) from DFT simulations) for estimating barriers (energies for Rh-doping case). The $\mathrm{Rh}, \mathrm{N}$ and $\mathrm{H}$ atoms are represented by purple, blue and red balls, respectively. Bronze, dark and white spheres represent the top, the second, and the third layer Fe atoms, respectively. To represent the complexity of various doping sites, we use different symbols here for adsorbed species than Figure 1 and 4. The symbol (1) represents the linear $2 \mathrm{~N}$ 2 $\mathrm{H}$ [linear2] structure while the symbol c (or f) represents a doping element put close to (or far from) the $\mathrm{N}, \mathrm{H}, \mathrm{NH}_{2}$ and $\mathrm{NH}_{3}$ species. 
Figure 4. The stardard state energy landscape for $\mathrm{NH}_{3}$ synthesis reactions on $\mathrm{Rh}$-doped $\mathrm{Fe}(111)$ surface under $673 \mathrm{~K}$ and $20 \mathrm{~atm}$ conditions. The lowest energy state $3 \mathrm{~N}_{-} \mathrm{NH}_{2}$ is taken as reference, with a free energy of zero. Black is the linear pathway and orange is the alternative pathway from the optimum reaction barriers. The notations for the adsorbed species represent the key configurations (Figure S4 of SI) along the reaction path. To be consistent with Figure 1, we used the same symbols for adsorbed species. 
Table 1. Barriers of rate-determining steps in ammonia synthesis over pure and doped $\mathrm{Fe}(111)$ surface estimated via the Brønsted-Evans-Polanyi (BEP) principle as discussed in the text. Barrier-5 corresponds to the maximum of barrier(1-4) plus the stability penalty term. Rightmost column is the expected $\mathrm{NH}_{3}$ production rate per $(2 \times 2)$ unit cell per second. Free energies in $\mathrm{eV}$.

\begin{tabular}{lllllll}
\hline Element & barrier-1 & barrier-2 & barrier-3 & barrier-4 & barrier-5 & Rate( $\left.\mathbf{S}^{-\mathbf{1}} \mathbf{6 7 3} \mathbf{~ K}\right)$ \\
\hline $\mathrm{Rh}$ & 1.51 & 1.60 & 1.31 & 1.44 & 1.60 & 14.61 \\
$\mathrm{Pd}$ & 1.51 & 1.63 & 1.42 & 1.51 & 1.63 & 8.71 \\
$\mathrm{Pt}$ & 1.55 & 1.59 & 1.36 & 1.60 & 1.60 & 14.61 \\
$\mathrm{Cd}$ & 1.55 & 1.84 & & & & \\
$\mathrm{Au}$ & 1.57 & 1.82 & & & & \\
$\mathrm{Co}$ & 1.58 & 1.51 & 1.42 & 1.45 & 1.76 & \\
$\mathrm{Ag}$ & 1.59 & 1.81 & & & & \\
$\mathrm{Ni}$ & 1.60 & 1.39 & 1.48 & 1.53 & 1.68 & \\
$\mathrm{Cu}$ & 1.64 & 1.61 & 1.56 & 1.52 & 1.64 & 7.33 \\
$\mathrm{Zn}$ & 1.64 & 1.79 & & & & \\
$\mathrm{Ir}$ & 1.65 & 1.88 & & & & \\
$\mathrm{Ru}$ & 1.67 & 1.76 & & & & \\
$\mathrm{Fe}$ & 1.68 & 1.57 & 1.53 & 1.43 & 1.68 & 3.68 \\
$\mathrm{Os}$ & 1.73 & & & & & \\
$\mathrm{Mn}$ & 1.73 & & & & & \\
$\mathrm{Ce}$ & 1.74 & & & & & \\
\hline
\end{tabular}




\section{TOC Graphic}

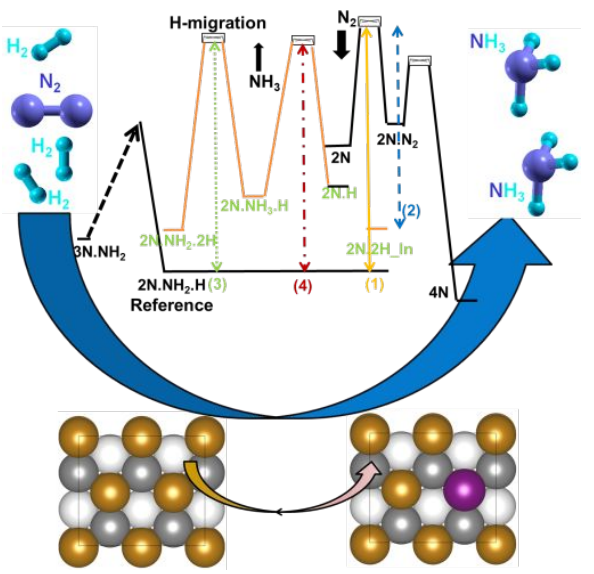

